\title{
Experiences of New Mothers with Premature Babies in Neonatal Care Units: A Qualitative Study
}

\author{
Gul Pinar ${ }^{*}$ and Hulya Erbaba* \\ Department of Nursing, Faculty of Health Science, Yildirim Beyazit University, Ankara, Turkey
}

\begin{abstract}
Background: Although mother-newborn attachment is very important for infant development babies in need of neonatal intensive care have to be separated from their mothers. A mother might experience distress in bonding with her vulnerable newborn because of admission to the neonatal care unit (NICU).

Purpose: The purpose of the study was to describe the new mothers' experiences of having a premature baby at the NICU.

Methods: This study was patterned with a descriptive qualitative design. Data were collected through in-depth interviews with 25 new mothers using semi-structured questions. Participant's each word has been recorded. Records were transcribed and content analysis was made.

Results: Four main themes and sub-themes were emerged regarding the experiences of mothers; 1) The barriers of having a premature newborn; feeling of separation from their baby, feeling of isolation towards NICU setting, feeling of emotional distress, 2) Lack of self-confidence for motherhood, 3) Maternal-newborn interaction, 4) Maternal-health staff interaction; insufficient communication and psychosocial support. Mothers stated that they felt themselves incompetent in terms of post-NICU care due to anxiety, inadequate support and self-confidence. They needed effective information about the hospital practices and post-NICU care.
\end{abstract}

Conclusion: Mothers presented many feelings with some positive and negative experiences such as sadness, alienation, fear and anxiety, depression, guilty, losing their self-confidence about maternity skills, and insufficient bonding with their babies. Mothers also expressed their needs and expectations as emotional support and empathic approach from staff. It is extremely important for mothers to get psychological support in order to cope with the infants staying in NICU.

\section{Keywords}

Prematurity, Neonatal intensive care, New-mothers, Maternal experience

\section{Introduction}

Preterm birth is a potentially stressful, an unexpected and a multi-problematic event such as neonatal respiratory problems, jaundice, hypotermia, feeding problems and infections [1-4]. After preterm delivery, as newborn need instantly stabilization (support respiratory and body term regulation etc.), they are generally admitted to the neonatal intensive care units (NICUs) $[3,5]$. Keeping mothers away from the NICU for reasons such as infection, noise, and privacy prevents mothers from getting closer to their babies $[1,4,6,7]$. Sophisticated equipment and continuous sound stimulation in NICU can impair the parental affective attachment process, and can be commonly terrifying for parents in the early postpartum period [8-10]. Previous studies have been reported that interrupted by sudden unexpectedly early delivery caused negative feelings on the including, confusion, anger, guilt, sufferance, weakness, tiredness, helplessness, frustration, depression, anxiety, lack of confidence, stress and loss of control, and experience feelings of inclusion and exclusion regarding the care of their babies $[8,11-15]$. Forcada-Guex, et al. [16] and Bonacquisti, et al. [12] explained that for mother-baby attachment and baby development, mother and baby should spend a lot of time together right after birth. However, in many clinical settings, there remains some reluctance to im-

*Corresponding author: Assoc. Prof. Gul Pinar, Department of Nursing, Faculty of Health Science, Yildirim Beyazit University, Ankara, Turkey;

Assist. Prof. Hulya Erbaba, Department of Nursing, Faculty of Health Science, Yildirim Beyazit University, Ankara, Turkey

Accepted: November 02, 2020

Published online: November 04, 2020

Citation: Pinar G, Erbaba H (2020) Experiences of New Mothers with Premature Babies in Neonatal Care Units: A Qualitative Study. J Nurs Pract 3(1):179-185 
plement this approach for newborns and preterm babies. To address this gap, evaluation of experiences faced by parents about parent-infant separation during neonatal care is an important need in order to better understanding of their challenges and also women's expressions can help us explore the best way to prepare for the child care period.

\section{Purpose}

The aim of the present study was to investigate new mothers' experiences in order to better understanding of their experiences during their premature infant's hospitalization at the NICU.

\section{Methodology}

\section{Research design, sampling and setting}

This qualitative study was performed between Jan and May 2016 in a tertiary hospital in Ankara, Turkey. Participants consisted of mothers whose babies were hospitalized at NICU. The mothers were selected by convenience sampling. A total of 25 mothers volunteered and consented to participate. The study hospital NICU has 29 beds (17 fully equipped, 2 isolation, 10 open incubators). Family-centered care policy has been adopted and implemented in the unit. As the inclusion criteria: 1 ) Mothers who gave birth before the $37^{\text {th }}$ gestational week (singly), 2) Whose babies were hospitalized in NICU for at least 3 days, and 3) Who could speak Turkish were preferred. Exclusion criteria were as follows: 1) Mothers whose children have died, 2) Mothers whose child had severe disability, and 3) Mothers who have spent $<3$ days at the hospital (the hospitalization period of $>3$ days was accepted as sufficient time for mothers to create "beneficial" experiences of NICU to share with us).

\section{Data collection}

The questions asked to the participants were directed using questionnaire form and also in-depth interviews were conducted using semi-structured questionnaires by the researcher. These interviews were held in a private room in the hospital's mother hotel. During the interviews; open-ended questions were used to identify the support elements received by healthcare professionals, and to explore mothers' feelings of having a preterm baby, and having to be hospitalized in the NICU. The open-ended questions included:

1. How do you describe your experience of their newborn's stay at the NICU? Please tell me about your experiences of taking care of your baby at NICU?

2. How did you feel initially after the infant's birth? Please can you describe your experiences when you first saw your baby?

3. How much do you think you have control of your baby's care? How do you evaluate your communication and connection with your baby?"

4. How was your communication with the healthcare staff? How NICU staff/ relatives approached your needs as the mother of a preterm baby. What more support would you like to receive in addition to these?
These interviews lasted between 30-40 minutes. During the interviews, the researchers not interrupted talking of the participants, and the talks continued until the participants repeat the same statements, and there was nothing different to say. The interviews were recorded and transcribed with a tape recorder within the knowledge of the participants.

\section{Data analysis}

Qualitative content analysis was used to classify concerning mothers' descriptions and feelings of their experiences using general thematic cods. The interviews listened repeatedly to gain a sense of the content by two researchers and also an independent women health nursing department's academician not involved in the study to comply with the impartiality and openness principle. Then transcribed categorically to capture the similar mothers' statements regarding their child's companion at the NICU.

\section{Ethical considerations}

Rules specified in the Helsinki Declaration were observed in the data collection phase. The study protocol was approved by the Ethics Committee of University hospital. Before the interviews, it was highlighted to the mothers that this study was a scientific study and would not directly benefit them today, but this information would be useful for mothers who had the same problem in the future. Mothers voluntarily provided written informed consent was obtained before study.

\section{Results}

The average age of the mothers was $27 .(20-42)$, the average age of gestational age was 26 weeks (24-28), and the average of hospitalization time was 2 weeks (1-4). The largest proportion of women had unemployed (78\%), had medium economic level (71\%), and had completed high school (73\%). All of mothers had health insurance.

The findings demonstrate that four main themes and their subthemes were identified:

\section{The emotional and psychological difficulties of having a premature newborn}

Feeling of separation-alienation from their baby: Mothers felt some challenges due to clinically unstable and the impairment of their premature child. Mothers described the preterm birth as a traumatic event that could increase stress and negative feelings such as sadness, separation, fear, anger, insecurity, anxiety, and depression. Among the interviewees who mentioned these feelings, the following stand out:

"At first when I saw my baby. I was in shock and scared about the appearance of my little baby. He was so tiny baby. I was afraid how can I hold such a very fragility baby?, He might be injured. It was difficult to carry of him properly. I felt incapable of taking care of my baby on my own and So, I could not actively involved in my baby' care at first time".

"When I first saw my baby in the incubator, I felt very sad. What was happening to my baby? I was so afraid to hurt him that I definitely shouldn't have touched him, I thought he just need grown up immediately. Otherwise I can't even breast- 
Citation: Pinar G, Erbaba H (2020) Experiences of New Mothers with Premature Babies in Neonatal Care Units: A Qualitative Study. J Nurs Pract 3(1):179-185

feed him. I was wondering that whether my baby will live any complication or not".

"I loved her more when she was in my womb. I feel sorry for her as a suffering being, but as if she is not my baby. I should be tied to her someway. I am afraid that my baby will be cut from the breast and away from me. If I can't breastfeed, I can't feel that it's mine".

"My baby is crying often, I can do nothing but watch it. I have a hard time holding it in my arms, I couldn't feel that I was a mother, I am afraid of not being able to love it, I am afraid that this feeling will deepen in me."

"I am always afraid to accept her as sick. I am very sad. My husband thinks that I am very panic about the condition of our baby, this approach makes me feel inadequate".

Feelings isolation towards the NICU setting: In the early period after the baby's admission to the unit, mother felt some difficulties; the attention of the mother focused on the NICU environment, and the baby remained in the second plan until they perceive the environment. Among the interviewees who mentioned these feelings, the following stand out:

"The complexity and loud sounds of the tools here scare me. A cable comes out from everywhere. Everywhere is like electric. My baby constantly hears these annoying sounds".

"Actually, the environment of the NICU is very stressful for me. I have never seen like this place before; doctors, nurses are constantly on the move. I worry if they mix things up. I am trying to be calm and positive, I prayed a lot, but I am not sure that I can easily bond with my baby".

"This unite is a warm, narrow and crowded. Special clothes are worn each time in this place; of course as it should be, but always busy people working there, it scares me. I feel hopeless about my baby's condition".

"The torment begins as I enter the door to breastfeed. Mask, cap, protective apron, hand disinfectant, different incubators, lights, sounds, drug scents ... materials attached to the my baby ... I want to cry, goose bumps, my nipple is pulled from stress."

"When I saw my little baby in the NICU, my initial reaction was very horrified. I was thinking about my baby as weak and in danger. He was apathy. He had tubes and wires coming out of every part of him. I felt astonished and then I started to cry."

Feelings of emotional distress: Mothers described "having a premature birth" as a very stressful and difficult transition period start especially for new mothers. Among the interviewees who mentioned these feelings, the following stand out:

"Always getting permission from someone to get to her is upsetting my nerves, they don't let me do anything. I cry all the time. I'm afraid I will get depressed. If my economic situation were good, my baby would develop better. I could not take care of myself enough. I could not feed it enough. She had a developmental delay, because of me."
"The irregular breathing and exhausted appearance of my baby scares me. I can't hold myself. I want to take it away. I wish I was sick if my baby couldn't breathe."

"Mothers are always confused and fearful, this affects me more than anything else. Mothers should be relieved. We cannot be left without this much knowledge. I want to ask someone to account for this and shout at them."

"I was working even at night in the private sector. I could not tell anyone about the risk of preterm birth, so as not to lose my job. Also, I regret I smoked during pregnancy, eat sweet-salty nutrient, and all kinds of wrong moves made this baby. I am usually anxious, seeing my baby in this environment brings me a lot of fear".

\section{Lack of self-confidence}

Mothers have stated concerns about their maternity role and having premature babies. They didn't have a chance to complete their baby's routine preparations before the baby's birth, which made it difficult for them to experience the feeling of motherhood after the baby's sudden birth. The following stand out:

"I am afraid of not being able to take good care of my baby and causing harm. "If I stay at the hospital until the baby grows a little, I will be prepared to breastfeed and care of him. Now, I'm so scared that they'll give me this baby before it gets better. I'm worried that if his situation continues and I can't understand, something bad will happen."

"What if I can't care for this baby, if I don't have enough patience, I do not feel comfortable yet when caring and breastfeeding her. I get depressed. How will I understand if he has a problem? Also, I worry about others criticizing my motherhood. I want my husband and his relatives to admire my motherhood."

"The bad news I heard from the media scares me. I am very afraid that if something runs into her throat, I fall asleep while breastfeeding. I crush the baby and get it out of bed. Why couldn't I take better care of my baby? I could feed her better. I must have done something missing."

"I have no self-confidence about baby care, if I can't handle it, I'm afraid of leaving or losing control enough to inflict violence on him. I have done this to my pet before. But this is a baby and I gave birth to it, I'm so confused, I need help."

\section{Maternal-newborn interaction}

It was determined that the more positive feelings the mothers had towards their babies, the less their initial worries. Mothers wanted to be close to the infant and hold him/ her as soon as possible after the birth. Among the interviewees who mentioned these feelings, the following stand out:

"At first I was afraid of my baby, but over time I changed, I accepted him. Now I have managed to go to intensive care and take him in my arms without fear. I miss him as soon as he leaves him. He is a part of me and I love him very much. My fear and anxiety gradually decreased."

"This situation was difficult to handle. Everything happens 
so quickly. Initially, I did not feel like a mother after my birth because I was not prepared yet. I need to spent time with him. I hope day by day I will be reinforced for care of my baby".

"I felt concern how I become a mom to my baby at the beginning. It was very stressful in connection with a preterm birth. I try to keep this unexpected situation under control because I should be strong".

"I was so shocked when I first visit the NICU. She was so tiny. She looked not very well in the incubator; she has many drips with feeding tube and many other things on their chest. I felt failed myself in motherhood and scared deeply then I started the cried."

"I'm going to take care of my baby alone when I get back home. I was thinking it's important to get closer contact with my baby for our affective bonds between us. Although I was very eager to take care of my baby, I could not touch her depending on my baby's clinical situation. Now, I felt strain".

"I went to the NICU for breastfeeding. She seemed to very in a vulnerable position at first. It makes me very sad to see my baby connected to the devices in intensive care. I was trying to connect to my baby and now our bonding is better, I did feel close to her."

\section{Maternal-health provider's interaction and ex- pectations}

Effective communication: The mothers were expressed their expectations from health professionals as encouraging to take care of their babies. Mothers wanted to get to know their babies by seeing, caring, holding, grasping, touching, and talking them as without interrupt as possible in the NICU. They also desired adequate information on their own nutrition, mental and physical recovery, breastfeeding, and motherhood, and encouragement to cope with their baby's care problems from NICU staff. Unfulfilled expectations made mothers feel both disappointment and sadness. These findings emerged in the following statement from mothers:

"I desired that involving in feeding my baby, changing nappies or playing with my babies to raise my maternal confidence". As I spent time at NICU, my distress gradually subsided and I adapted better to my baby care routines. I need someone to support me".

"My first encounter with my baby was much more difficult than I thought. We could not spend all time at the hospital. I did not leave my baby in there. But there was nothing I could do. Then I tried to focus more realistic balance with the support of staff".

"I was sometimes even depressed but health providers in the NICU who provide constantly informed about our baby' condition and all procedures care for the baby, they assisted how should I hold my baby. I always trust them because they stay very close to our baby thereby this guidance is very crucial for me".

"It is very difficult to communicate with the healthcare team. I had trouble getting used to the situation because of their attitude. Not everyone is the same, of course, but I have seen that nurses work hard and cannot reach babies. Doctors also work hard, so everyone. Babies are small, a lot of work here. After all, nobody gives enough information and I have no one to help."

"Nurses are different, I am afraid to ask questions every time. Also they touch the baby as if it were an item, some try to help, some are asking me to ask the doctor. Doctors make explanations, but their work shows that they are bored with me. They are trying to help but not enough for me. In general, they are not/cannot be supported."

"In fact, when it's time to breastfeed, nurses call me right away. They always allowed me to breastfeed my baby. I was able to breastfeed day and night, which was very good. I feel my baby safe when they explain to me. They treat me even closer because I will give the baby to others."

Psycho-social support: The majority of both groups of participants reported that providing mothers with psychosocial support was crucial for facilitating maternal-newborn bonding. These findings revealed in the following statement from mothers:

"I would expect obstetricians to come to us to explain some issues. For example, my breast is swollen, my stitches hurt, I had a cesarean vaginal bleeding continues violently. Will I go to the doctor for everything?"

"I felt more relaxed going to the NICU and to receive update nurses about any progress, also I could participate fully to care for my baby, I could feed my baby and I could ask any questions for the development of my motherhood. "Even though everyone and opportunities are good, my psychology is broken. I would like psychologists or social workers to ask our attention."

"I was able to manage my confused about separation with my baby because I had regular have information regarding my baby's situation. Having a contact person was very helpful. Now, health providers' interactions become less anxious for me".

"Healthcare providers helped and counseled us psychologically. I can express my concerns toward them. Mother hotel is a very good practice. I feel secured and satisfied now about my child's condition."

"I was able to stay in the hospital hotel, this is a very nice opportunity. Nurses turned to with my questions. It is a very positive thing that there is a woman employee who meets the needs of women here."

\section{Discussion}

In the present study, mothers listed problems as a barrier such as not being able to breastfeed the baby, not being able to establish intimacy with their baby, and not being able to sufficient communicate with healthcare professionals. Mothers stated that they have difficulties in meeting vital needs such as facilitating the early mother-infant relationship, solving their strain and concern in early stages following the birth of their baby. Beyond that, they explained the inadequacy of socio-psychological support as "later problems", although 
they are very important. The findings of previous studies are similar to our results, also mothers described hospitalization and discharge procedures and social security as an additional barrier issues $[10,17,18]$.

In a study performed by Jackson, et al. [19] mothers have felt in control during pregnancy. For them, the baby was the happiness element of life after birth. Suddenly handing over this source of happiness to other hands, due to a health problem, drove them away from their babies. In this study, the emotions that make mothers feel alienated from their babies; the delicate and fragile nature of babies, difficulty in touching, difficulty in breastfeeding or expressing milk in difficult conditions for her, and not being able to participate in the care of the baby as a mother. They were concerned that the preterm infant would be affected for survival. Furthermore, mothers consider the experience of having their baby admitted to a NICU as distressing and traumatic, and life altering. Similar results have been demonstrated in other studies $[10,13,20,21]$.

Both "bonding" and "attachment" relationship develops better with the continuous union of mother and baby. However, normal bonding process is hindered by illness, NICU hospitalization after premature births may cause psychological and social problems in mothers. Therefore, understanding NICU mothers' psychosocial needs is important also to contribute to the best possible long- term outcome for the infant and family [22]. The rapid alienation of this mother from her baby supports this view and reveals the difference between the other mothers and this mother withdrawing from their babies [23]. In the present study, mothers explained that they were afraid of the complex environment in which these advanced techniques were used and that they were surprised by the specialization of the doctors and nurses. On the other hand, they were worried about the disruption of the treatment of their babies among such complex procedures. Studies have shown that the technical equipment and the medical language spoken in NICUs all over the world cause stress in mothers and they feel very lonely here [5,8,11-13]. In different studies, it was revealed that mothers experienced emotional distress; some blame themselves, some feel so tense, and some want to eliminate the factors that prevent her from reaching her with a very high anxiety for her baby $[7,19,24]$. In a meta-analytic study, mothers stated that they are highly affected by all kinds of stressors during their hospital stay, this situation differs according to the severity of the baby's health problem and the mother's previous psychological problems, especially the first week of the postpartum period is the period with the highest stress. In the same study, mothers cannot cope with baby's care, depressive, and not being able to control her emotions [25].

In the present study, mothers basically stated that they felt inadequate about baby care. These shortcomings; the thought of proving oneself as a good mother to others, anxiety of not being able to cope with difficulties and running away, anxiety about leaving the baby or the environment, and not being able to notice possible problems with the baby due to inexperience have been expressed. The sudden introduction of a baby to intensive care after birth causes mothers to decrease their self-efficacy [26]. Studies have shown that mothers with poor economic status, poor family communication, unmarried, and seriously premature babies (low birth weight, premature, anomaly) have low self-confidence $[27,28]$.

Quality togetherness of mother and baby is important for a healthy mother-infant attachment [11]. It has been stated in previous studies that babies develop mentally and physically faster as mothers have more opportunities to be with and care for their babies $[20,22,26]$. In this study, mothers; it was revealed that they could not touch their babies enough, could not hold them, talk to them, spend time together and this situation strained the mother. According to the studies, the NICU environment has created a barrier to mother-infant interaction and it was also explained that health professionals play a facilitating role in mother-infant interaction $[12,29,30]$.

Expectations of mothers in this study; care for this particular baby is disclosure of his condition and information and attention to intimacy. Some mothers found the support of healthcare professionals very positive. NICU services in Turkey have been deployed in the overall teaching and research hospital. The complexity of these units and the workload of the nurses may not allow these professionals to adequately interact with the mother. In another study where the two groups were compared, nurses provided education and social support for mothers' babies remaining in NICU. It was observed that some mothers suffered from nurses' indifference, some mothers got stronger by receiving staff support [29]. Furthermore, another study reported that nurses and doctors do not fulfill their educational roles and are even perceived as irresponsible. Mothers expected heath providers to be more directive and friendly [23].

The findings of this study revealed that the emotional states of mothers in NICU processes are generally sensitive. Mothers have high concerns on many issues, especially the insufficient maternal involvement and fear of losing their baby. In this study, "communication" could be a lifeline for them to deal with their problems. In this study, mothers mostly stated that their expectations about communication were not met. A few of them participated in the care through nurses and this experience reduced the anxiety of the mothers. The mother hotel was considered as a very good opportunity as it allows mothers to stay and get closer to the baby. This opportunity was found positive in the study. Mothers also reported that a psychologist should take care of them for mental support and a social worker for their social needs. Similarly, previous studies reported that mothers were unable to deal with doctors at all, and they complained that communicating with health staff was very difficult and they used negative communication methods, which pushed a mother to loneliness. Although communication skills differ from person to person, it is thought that the communication of healthcare professionals with mothers, even at a basic level, will ensure the adaptation of mothers to NICU and reduce the workload of these professionals $[5,11,23,24,29,30]$.

\section{Conclusion and Recommendations}

The feelings and needs of mothers with preterm baby in 
the NICU were varies from mothers to mothers in this study. Mothers especially needed to be enhancing their maternal role. According to the mothers, health providers in the NICU was essential role for accomplish early maternal-newborn bonding. In this regard, mothers must be given adequate support by health staff in order to solve their emotional problems and ensure early bonding with their babies. Further and larger comprehensive studies are needed to explore mothers' feelings and to implement family-centered interventions in order to enhance mother-newborn interactions in the NICU.

\section{Limitation}

This study was performed in the NICE of tertiary hospital. For this reasons, the findings may not represent of all hospitals in general.

\section{Conflict of Interest}

The authors declare no conflict of interest.

\section{Authors' Contributions}

$\mathrm{HE}$ and GP planned and designed the study. HE performed the interviews. GP analyzed the data and wrote the manuscript. Both authors read and approved the final manuscript.

\section{Acknowledgement}

The researchers are grateful to the management of hospital for allowing the researchers entry as well as all the mothers for their contributions.

\section{Funding}

The authors received no financial support for the research, authorship and/or publication of this article.

\section{References}

1. Polin RA, Denson S, Brady MT, et al. (2012) Committee on infectious diseases. Epidemiology and diagnosis of health care-associated infections in the NICU. Pediatrics 129: e1104-e1109.

2. Alhusen, JL, Hayat MJ, Gross D (2013) A longitudinal study of maternal attachment and infant developmental outcomes. Arch Women's Mental Health 16: 521-529.

3. Parkash A, Haider N, Khoso ZA, et al. (2015) Frequency, causes and outcome of neonates with respiratory distress admitted to neonatal intensive care unit, national institute of child health, Karachi. J Pak Med Assoc 65: 771-775.

4. Cheong JL, Burnett AC, Treyvaud K, et al. (2020) Early environment and long-term outcomes of preterm infants. J Neural Transmission 127: 1-8.

5. Ncube RK, Barlow H, Mayers PM (2016) A life uncertain - My baby's vulnerability: Mothers' lived experience of connection with their preterm infants in a Botswana neonatal intensive care unit. Curationis 39: e1-e9.

6. Valizadeh L, Akbarbeglou M, Asad EM (2009) Stressors affecting 636 mothers with hospitalized premature newborn in NICUs of three teaching hospitals in Tabriz. Med J Tabriz Univ Med Sci 31: 85-90.

7. Goutaudier N, Lopez A, Séjourné N, et al. (2011) Premature birth: Subjective and psychological experiences in the first weeks following childbirth, a mixed methods study. J Reprod Infant Psychol 29: 364-373.

8. Ionio C, Blasio DP (2014) Post-traumatic stress symptoms after childbirth and early mother-child interaction: An exploratory study. J Reprod Infant Psychol 3: 163-181.

9. Beck CT, Harrison L (2017) Posttraumatic stress in mothers related to giving birth prematurely: A mixed research synthesis. J Am Psychiatr Nurses Assoc 23: 241-257.

10. Klawetter S, Neu M, Roybal KL, et al. (2019) Mothering in the NICU: A qualitative exploration of maternal engagement. Soc Work Health Care 58: 746-763.

11. Nazari R, Moradi Koosha F, Rezaie S, et al. (2020) Experiences of the mothers of infants hospitalized in the neonatal intensive care unit (NICU). J Neonatal Perinatal Med 1-9.

12. Bonacquisti A, Geller PA, Patterson CA (2020) Maternal depression, anxiety, stress, and maternal-infant attachment in the neonatal intensive care unit. J Reprod Infant Psychol 38: 297-310.

13. Maguire CM, Bruil J, Wit JM, et al. (2007) Reading preterm infants' behavioral cues: An intervention study with parents of premature infants born $<32$ weeks. Early Hum Develop 83: 419424.

14. Grunberg VA, Geller PA, Bonacquisti A, et al. (2019) NICU infant health severity and family outcomes: A systematic review of assessments and findings in psychosocial research. J Perinatol 39: 156-172.

15. Doede M, Trinkoff AM, Gurses AP (2018) Neonatal intensive care unit layout and nurses' work. HERD 11: 101-118.

16. Forcada-Guex M, Borghini A, Pierrehumbert B, et al. (2011) Prematurity, maternal posttraumatic stress and consequences on the mother-infant relationship. Early Hum Dev 87: 21-26.

17. Malakouti J, Jabraeeli M, Valizadeh S, et al. (2013) Mothers' experience of having a preterm infant in the Neonatal Intensive Care Unit, a Phenomenological Study. Iranian J Crit Care Nurs 5: 172-181.

18. Raffray M, Semenic S, Osorio Galeano S, et al. (2014) Barriers and facilitators to preparing families with premature infants for discharge home from the neonatal unit. Perceptions of health care providers. Invest Educ Enferm 32: 379-392.

19. Jackson K, Ternestedt BM, Schollin J (2003) From alienation to familiarity: Experiences of mothers and fathers of preterm infants. J Adv Nurs 43: 120-129.

20. Jouybari LM, Haghdoost Oskouee SF, Ahmadi F (2005) Comforting nurse: Patients' experiences and perceptions. Razi J Med Sci 12: $59-68$.

21. Kohan M, Borhani F, Abbaszadeh A, et al. (2012) Experience of mothers with premature infants in neonatal. J Qual Res Health Sci 1: 41-51.

22. Ngaiyaye EP, Kalembo FW (2016) Supporting mothers to bond with their newborn babies: Strategies used in a neonatal intensive care unit at a tertiary hospital in Malawi. Int J Nurs Sci 3: 362-366.

23. Holditch-Davis D, Miles MS (2000) Mothers' stories about their experiences in the neonatal intensive care unit. Neonatal Network 19: 13-21.

24. Pace CC, Spittle AJ, Molesworth CM, et al. (2016) Evolution of depression and anxiety symptoms in parents of very preterm infants during the newborn period. JAMA Pediatr 170: 863-870. 
25. Staver Morgan A, Tiffany A Moore, Kathleen M Hanna (2020) An integrative review of maternal distress during neonatal intensive care hospitalization. Arch Women's Mental Health 1-13.

26. Vance AJ, PanW, Malcolm WH, et al. (2020) Development of parenting self-efficacy in mothers of high-risk infants. Early Hum Dev 141: 104946.

27. Sevigny PR, Loutzenhiser $L$ (2010) Predictors of parenting self-efficacy in mothers and fathers of toddlers. Child Care Health Dev 36: 179-189.
28. Salonen AH, Oommen H, Kaunonen M (2014) Primiparous and multiparous mothers' perceptions of social support from nursing professionals in postnatal wards. Midwifery 30: 476-485.

29. Tandberg BS, Frøslie KF, Flacking R, et al. (2018) Parent-infant closeness, parents' participation, and nursing support in single-family room and open bay NICUs. J Perinat Neonatal Nurs 32: E22-E32.

30. Treyvaud K, Spittle A, Anderson PJ, et al. (2019) A multilayered approach is needed in the NICU to support parents after the preterm birth of their infant. Early Hum Dev 139: 104838. 